\title{
Mindfulness-based cognitive therapy (MBCT) versus the health-enhancement program (HEP) for adults with treatment-resistant depression: a randomized control trial study protocol
}

Stuart J Eisendrath ${ }^{1 *}$, Erin P Gillung ${ }^{1}$, Kevin L Delucchi ${ }^{1}$, Maggie Chartier ${ }^{2}$, Daniel H Mathalon ${ }^{1,2}$, Jude C Sullivan ${ }^{3}$, Zindel $V$ Segal ${ }^{4}$ and Mitchell D Feldman ${ }^{5}$

\begin{abstract}
Background: Major depressive disorder (MDD) is the leading cause of disability in the developed world, yet broadly effective treatments remain elusive. Up to $40 \%$ of patients with depression are unresponsive to at least two trials of antidepressant medication and thus have "treatment-resistant depression" (TRD). There is an urgent need for cost-effective, non-pharmacologic, evidence-based treatments for TRD. Mindfulness-Based Cognitive Therapy $(M B C T)$ is an effective treatment for relapse prevention and residual depression in major depression, but has not been previously studied in patients with TRD in a large randomized trial.

Methods/Design: The purpose of this study was to evaluate whether MBCT is an effective augmentation of antidepressants for adults with MDD who failed to respond to standard pharmacotherapy. MBCT was compared to an active control condition, the Health-Enhancement Program (HEP), which incorporates physical activity, functional movement, music therapy and nutritional advice. HEP was designed as a comparator condition for mindfulness-based interventions to control for non-specific effects. Originally investigated in a non-clinical sample to promote stress reduction, HEP was adapted for a depressed population for this study. Individuals age 18 and older with moderate to severe TRD, who failed to respond to at least two trials of antidepressants in the current episode, were recruited to participate. All participants were taking antidepressants (Treatment as usual; TAU) at the time of enrollment. After signing an informed consent, participants were randomly assigned to either MBCT or HEP condition. Participants were followed for 1 year and assessed at weeks 1-7, 8, 24, 36, and 52. Change in depression severity, rate of treatment response and remission after 8 weeks were the primary outcomes measured by the clinician-rated Hamilton Depression Severity Rating (HAM-D) 17-item scale. The participant-rated Quick Inventory of Depression Symptomology (QIDS-SR) 16-item scale was the secondary outcome measure of depression severity, response, and remission.
\end{abstract}

Discussion: Treatment-resistant depression entails significant morbidity and has few effective treatments. We studied the effect of augmenting antidepressant medication with MBCT, compared with a HEP control, for patients with TRD. Analyses will focus on clinician and patient assessment of depression, participants' clinical global impression change, employment and social functioning scores and quality of life and satisfaction ratings.

Trial registration: ClincalTrials.gov identifier: NCT01021254

Keywords: Depression, Treatment-resistant, Mindfulness, Meditation, MBCT

\footnotetext{
* Correspondence: StuartE@lppi.ucsf.edu

'University of California, San Francisco Department of Psychiatry, 401

Parnassus Avenue, Box 0984-AIP, San Francisco, CA 94143, USA

Full list of author information is available at the end of the article
} 


\section{Background}

Major depressive disorder (MDD) is the number one cause of disability in developed countries and is projected to become the number two cause of disability worldwide by 2020 [1,2], yet broadly effective treatments remain elusive. The World Health Organization (WHO) Primary Care Study found that $60 \%$ of patients treated with antidepressants still met criteria for depression after one year of treatment [1]. Treatment-resistant depression (TRD), the term used to describe cases of MDD that do not remit with adequate courses of at least two antidepressant trials [3,4], is unfortunately quite common [3,5-8]. The seminal Sequenced Treatment Alternative To Relieve Depression $\left(\mathrm{STAR}^{*} \mathrm{D}\right)$ study found that only $30 \%$ of individuals with MDD will remit with one full trial of antidepressant medication, and the remission rates for successive trials are even lower $[3,8]$. TRD is associated with greater disability, mortality, morbidity, somatic symptoms, risk of relapse and societal cost than those who suffer from non-resistant forms of depression $[4,9,10]$. Hence, there is an urgent need for innovative and effective treatments.

Mindfulness-Based Cognitive Therapy (MBCT) is a group-based, 8-week, mind-body intervention that integrates mindfulness meditation with concepts of cognitive behavioral therapy (CBT) and was specifically developed as a relapse prevention intervention for MDD [11]. MBCT's approach is distinctive, as it focuses on the cultivation of effective methods to relate to depressive thoughts and feelings, rather than challenging or changing specific cognitions as taught in CBT. MBCT teaches patients to relate to their unpleasant thoughts, feelings, and bodily sensations as temporary passing events in the mind, rather than identifying with them or treating them as accurate reflections of reality.

Several studies have investigated MBCT as an augmentative treatment for acute depression. As an augmentor to medications, results indicate that $\mathrm{MBCT}$ has similar efficacy to CBT [12,13]., MBCT augmentation was more efficacious than antidepressant management alone in reducing the residual symptoms of chronic depression $[14,15]$ and improved outcomes in dysthymia compared to imipramine monotreatment [16]. Open label trials of MBCT augmentation of antidepressants for TRD showed effect sizes of 1.0 (Cohen's D) in studies by both Kenny and Williams [12] and Eisendrath et al., [13], making the investigation of MBCT as a potentially effective augmentative treatment for TRD worth further examination in a full randomized controlled trial (RCT).

\section{Research objectives}

The primary objective of this trial was to evaluate whether Mindfulness-Based Cognitive Therapy is an efficacious treatment for reducing depressive symptoms in adults with TRD. Individuals with TRD may be excellent candidates for MBCT [12,17], yet previous RCTs evaluating MBCT for depression failed to include an active comparator condition, limiting the results by not controlling for the non-specific factors (e.g., group support, homework, and facilitator attention) potentially associated with MBCT efficacy. With this limitation in mind, an active control condition, originally developed to be a structurally equivalent, comparison condition for studies Mindfulness-Based Stress Reduction MBSR) the Health-Enhancement Program (HEP) was selected for this trial.

The study's secondary objectives were to assess whether MBCT would be more effective than HEP in reducing symptoms of functional disability and improving overall quality of life and satisfaction and to examine potential mediators of MBCT treatment response. Finally, the study aimed to evaluate whether MBCT's potential treatment effects related to depression severity, functional status, and mindfulness would be sustained during 1-year follow-up phase.

\section{Methods/Design}

This study is a single-site, randomized, controlled clinical trial of MBCT augmentation of Treatment-as-Usual $(\mathrm{MBCT}+\mathrm{TAU})$ and HEP augmentation of TAU $(\mathrm{HEP}+$ TAU). TAU consisted of standard medication management for depression. In a single-blind fashion, participants were aware of treatment allocation but were blind to study hypothesis. Research assistants (RA's) responsible for assessment of depression severity, were blind to individual randomization assignments. See Table 1 for a list of inclusion and exclusion criteria.

\begin{tabular}{|c|c|c|}
\hline Factor & MBCT & HEP \\
\hline Manualized intervention & $x$ & $x$ \\
\hline Mindfulness training & $x$ & \\
\hline Music therapy & & $x$ \\
\hline Nutrition education & & $x$ \\
\hline Group support & $x$ & $x$ \\
\hline Therapeutic attention & $x$ & $x$ \\
\hline Session time (135 minutes) & $x$ & $x$ \\
\hline Session duration (8 weeks) & $x$ & $x$ \\
\hline Physical activity & X (e.g. yoga) & $\begin{array}{l}X \text { (e.g. exercise, } \\
\text { stretching) }\end{array}$ \\
\hline Functional movement & & $x$ \\
\hline Time equivalent homework & $x$ & $x$ \\
\hline Facilitator buy-in to method & $x$ & $x$ \\
\hline
\end{tabular}

Note. $\mathrm{X}$ indicates the presence of each component in the intervention. 


\section{Description of interventions}

The two treatments, MBCT and HEP consisted of 8 weekly classes, each lasting 2.25 hours. In addition to attending weekly classes, participants in both groups were encouraged to complete 45 minutes of homework six days per week for the duration of the 8-week treatments. Both interventions were conducted in a group format with 6-12 participants per group and were led by two facilitators extensively trained in their respective techniques.

MBCT is a manualized treatment that combines training in mindfulness meditation with elements of cognitive therapy. For the purpose of this study, MBCT was adapted from the original manual developed by Segal et al. [11] with modifications for TRD, the details of which are published elsewhere [13]. These modifications are believed to promote enhanced emotional regulation specific to TRD through increasing an individual's nonjudgmental awareness of their present moment experience [13]. MBCT teaches skills that encourage patients to disengage from habitual ("automatic") dysfunctional cognitive routines, in particular depression-related ruminative thought patterns, as a way to reduce current symptoms, future risk of relapse and recurrence [16]. Through guided meditation practices and activities, participants are taught to develop more present-focused, non-judgmental awareness of their thoughts and feelings instead of focusing on the content of them $[11,14,15,18]$. It is theorized that the same cognitive processes known to make individuals prone to depression relapse are also active in perpetuating depression once it is established [12] and are a primary driver of TRD.

HEP was developed as a collaborative effort between the Waisman Laboratory for Brain Imaging and Behavior at the University of Wisconsin - Madison and the University of Wisconsin Hospital and Clinics Sports Medicine Center in consultation with the National Center for Complementary and Alternative Medicine (NCCAM). HEP was designed to provide health benefits to participants while omitting any components of mindfulness and was developed specifically as a control condition for studies of MBSR [19,20]. Originally used as a control condition for stress reduction in non-clinical samples, we adapted HEP to be an active comparison condition for individuals with TRD. HEP provides participants with intensive training in aerobic exercise (i.e. walking), functional movement, music therapy, and nutritional advice. Classes encourage participants to engage in experiential, didactic exercises which include exercise, functional movement, food journaling, song-writing, guided imagery and drawing. Participants are provided with psycho-educational lectures on topics related to aerobic exercise, novel functional movement patterns, nutrition, establishing a balanced diet, and how these health elements can impact mood.
Similar to the MBCT program, HEP participants were encouraged to individualize the program to meet their unique needs and symptoms. They were asked to attend all weekly sessions and engage in approximately 45 minutes of homework assignments on the remaining 6 days of the week. A portion of each class was devoted to participants describing their experience of the group and the "barriers" to treatment that arose in the completion of the formal and informal (i.e. homework) practices assigned by facilitators, which mirrors the same model used in MBCT. After the completion of the program, participants were provided with community resources to continue their practices, just as referral resources were provided during the final MBCT session.

In summary, HEP provides a credible comparator condition for MBCT by controlling for features of MBCT such as class participation, overall time invested, attention, group process, social interaction and support, homework, and experiential participation through in-class exercises (Table 1). A comparison of the course content by session for each intervention is outlined in Table 2. Study participants were not engaged in individual or group psychotherapy during the study treatment time period.

TAU augmentation for both treatment groups was identical and consisted of medication management and supportive counseling delivered by third or fourth year psychiatric residents, attending psychiatrists or primary care physicians. Supportive therapeutic approaches aid in patients addressing problems without direct input from providers. Providers offer patients encouragement and guidance but do not engage in any therapeutic strategy other than active listening and offering support by focus on patients' problems and concerns. Routine TAU visits are less than 30 minutes in duration and at intervals of one to three months. Participants were allowed to make medication changes during the acute and follow-up periods, with reporting of such changes on a regular basis. These changes will be incorporated into the analyses.

\section{Sample size}

To estimate sample size, effect sizes were based on outcomes from previous studies and our pilot data [15,21-24]. In a review of 21 mindfulness-meditation interventions, Baer [23] found an average effect size of .90 for depressive symptom reduction. In examining the relapse rates of individuals receiving MBCT versus TAU, Teasdale et al. [14] found an effect size of .59 and Ma et al. [15] of .88 for participants with a history of 3 or more depressive episodes. Our pilot data indicated an average effect size of 1.13, which compared similarly to Kenny's effect size of 1.03 in a TRD population [12]. Finucane et al., working with a more severely depressed population, found an effect size of 1.54 [25]. Kingston et al. found an effect size of 1.07 in reducing 
Table 2 Mindfulness-Based Cognitive Therapy (MBCT) and the Health Enhancement Program (HEP) Content by Session

\begin{tabular}{|c|c|c|}
\hline Session & MBCT & HEP \\
\hline 1 & Automatic Pilot: focuses on becoming aware in the present moment. & $\begin{array}{l}\text { Problems in Improving Health: focuses on physical fitness, functional } \\
\text { movement exercises, and music and imagery. }\end{array}$ \\
\hline 2 & $\begin{array}{l}\text { Dealing with Barriers: Participants discuss problems in focusing } \\
\text { attention and are encouraged to adopt an attitude of "letting go" } \\
\text { of self-criticism and judgment. }\end{array}$ & $\begin{array}{l}\text { Physical Activity to Enhance Well-being: Physical activities focus on } \\
\text { posture and alignment as ways to optimize function. Supportive } \\
\text { music imagery exercises are used to improve sense of well-being. }\end{array}$ \\
\hline 3 & $\begin{array}{l}\text { Mindfulness of the Breath: Participants complete a sitting meditation } \\
\text { in which they shift from focusing on body sensations to focus on } \\
\text { their breath in a sitting posture. Mindful movement techniques are } \\
\text { begun. }\end{array}$ & $\begin{array}{l}\text { Understanding Dietary Guidelines: Participants engage in a lecture } \\
\text { and discussion series about basic nutrition principals. Diet journaling } \\
\text { is reviewed and demonstrated as a way to better understand } \\
\text { healthy food choices. }\end{array}$ \\
\hline 4 & $\begin{array}{l}\text { Staying Present: Concepts of attachment/avoidance to pleasant/ } \\
\text { unpleasant sensations, moods, and thoughts are discussed. The } \\
\text { "three-minute breathing space" is taught as a simple intervention } \\
\text { for use throughout the day. }\end{array}$ & $\begin{array}{l}\text { Fine-tuning Dietary Choices: Participants learn the use of the USDA* } \\
\text { My Pyramid Food Tracker Program and behavioral changes are } \\
\text { discussed as a way to meet dietary goals. }\end{array}$ \\
\hline 5 & $\begin{array}{l}\text { Allowing/Letting Be: Focus is on allowing experiences "to be" } \\
\text { without judging or trying to change them. }\end{array}$ & $\begin{array}{l}\text { Adapting to an Unpredictable Environment- Part I: Activities focus } \\
\text { on functional movement and alignment. Supportive Music Imagery } \\
\text { focuses on responding to the environment in creative ways. }\end{array}$ \\
\hline 6 & $\begin{array}{l}\text { Thoughts Are Not Facts: Focus is on viewing thoughts as mental } \\
\text { events and not concrete facts. }\end{array}$ & $\begin{array}{l}\text { Adapting to an Unpredictable Environment - Part II: Activities such } \\
\text { as walking, jogging and stretching are discussed as participants are } \\
\text { encouraged to respond to the environment in creative ways to } \\
\text { enhance well-being. }\end{array}$ \\
\hline 7 & $\begin{array}{l}\text { How Can I Best Take Care of Myself: Participants learn their unique } \\
\text { warning signs of depression relapse or worsening. }\end{array}$ & $\begin{array}{l}\text { Responding to the Environment in Creative Ways: Participants learn } \\
\text { the kinetic chain of functional movement and build group cohesion } \\
\text { through group song writing exercises. }\end{array}$ \\
\hline 8 & $\begin{array}{l}\text { Using What Has Been Learned to Deal with Future Moods: } \\
\text { Participants discuss how they will keep the momentum of their } \\
\text { practice going. Therapists distribute information about settings } \\
\text { where group mindfulness practice can be continued. }\end{array}$ & $\begin{array}{l}\text { Support for Continuing Practice: Participants review the course and } \\
\text { plan for maintaining gains including all components of HEP. } \\
\text { Participants review their goals with the dietician.+ }\end{array}$ \\
\hline
\end{tabular}

*USDA = United States Department of Agriculture.

+ The original HEP protocol was amended to include Nutrition in session 8.

depression in psychiatric outpatients [26]. There were no significant differences in effect on depression in a comparison of HEP and MBSR [19,20]. Using the formula provided by Donner and Klar [27], we estimated sample sizes to detect response to the intervention (MBCT + TAU would be superior to HEP + TAU on depression outcomes). Results indicated sample sizes ranging up to approximately 50 per condition. A total sample of 124 participants were regarded as necessary to provide $80 \%$ power to detect a moderate effect size in the face with an estimated attrition of approximately $20 \%$, similar to our rates of attrition in clinical MBCT groups.

\section{Recruitment}

Study recruitment was conducted between September 2009 and September 2013 in outpatient psychiatry and general medicine clinics at the University of California, San Francisco (UCSF) Medical Center and the outpatient psychiatry clinic at Kaiser Permanente in San Francisco. Both recruitment sites were housed in large medical centers that serve an ethnically diverse population of privately and publically insured adults in the San Francisco Bay Area. To further contribute to the diversity of the patient sample, community locations were added to the recruitment plan at later stages in the study, as well as clinics at San Francisco General Hospital.
Recruitment methods were approved by the institutional review board (IRB) at the UCSF Human Research Protection Program (\#10-00455). For patients served through general medicine clinics at UCSF, approval was obtained to introduce the study with a letter from the principal investigator (PI) to prospective research subjects who had been identified through a query of patient medical record databases to have an ICD-9 code of depression and/or a medical history of taking antidepressant medication. Patients receiving the letter were invited to opt out of the study by mail within two weeks of receiving the letter. Patients who chose not to opt out were contacted by telephone by a RA to assess interest and eligibility to participate in the study. Potential candidates from other recruitment sites were referred by various methods, including research flyers, referrals from treating physicians, online announcements and print advertisements posted in public spaces and inside of buses and other public transportation vehicles in the Bay Area.

\section{Eligibility criteria (inclusion and exclusion criteria)}

Participants age 18 or older were required to meet DSMIV criteria for major depressive disorder and have a total score of 14 or higher on the clinician-rated, 17-item version of the Hamilton Depression Severity Rating Scale (HAM-D) [28] to be eligible for the study. To 
meet criteria for TRD, participants were required to be taking antidepressant medications with evidence of at least two adequate antidepressant trials prescribed during the current depressive episode. Participants were excluded (Table 3) from the study for the following reasons: they a) met DSM-IV criteria for bipolar disorder, obsessive compulsive disorder, or post-traumatic stress disorder (other comorbid Axis I conditions were noted but not cause for exclusion); b) had a history of schizophrenia or other psychotic symptoms; c) were imminently suicidal, a danger to others or were currently exhibiting self-injurious behavior; d) met DSM-IV criteria for alcohol or substance abuse or dependence within 3 months of study entry; e) were currently practicing meditation more than once per week or yoga more than two times per week; f) had cognitive impairment as evidenced by a score of $<25$ on the MMSE; g) were in concurrent individual or group psychotherapy and were not willing to discontinue treatment for the 8-week duration of study treatment; h) had significant or unstable medical illness that would limit participation in HEP; or i) did not have adequate English language comprehension.

\section{Assessment of eligibility and informed consent}

Interested individuals underwent initial screening for eligibility using a brief, structured telephone interview. Those with depressive symptoms being treated with antidepressants medications and not meeting exclusion criteria in Table 3 were invited for an in-person screening evaluation to determine eligibility and review informed consent. Psychological eligibility criteria such as depression severity and psychiatric history were assessed by a licensed mental health professional on the research team who had undergone intensive standardized training provided by one of the authors. Prior to the first assessment session, RAs explained the trial to participants by reviewing the study procedures, treatment interventions and required time commitment, and participants were given an opportunity to ask questions before signing informed consent. Participants were reminded that study participation was voluntary and that they had the right to withdraw from the research at any time without disruption to their usual care.

During the screening assessment, consenting participants provided information about their socio-demographic status. Assessment of depression severity and psychiatric history was conducted through a semi-structured clinical interview using the Structured Clinical Interview for DSMIV (SCID) [29], the HAM-D [28] and the Clinical Global Impression Severity (CGI-S) [30] scale. The SCID personality inventory for Axis II disorders (SCID-II) was used to assess the presence of personality disorders, and the Mini Mental Status Exam (MMSE) [31] was used to assess cognitive function and to rule out cognitive or developmental delays that might interfere with treatment. Treatment history and adequacy of antidepressant medication treatment was evaluated using the Antidepressant Treatment History Form (ATHF) [32]. Confirmation of at least 2 adequate trials of efficacy with a minimum total intensity score of 5 points or greater was required for study entry. In cases where treatment history or medication compliance was in question, RAs received informed consent from participants to review medical records and/or contact prescribing physicians to confirm adequacy of treatment. Finally, participants were asked to complete the self-report Cumulative Illness Rating Scale (CIRS)

\section{Table 3 Eligibility criteria for the PATH-D study}

\begin{tabular}{|c|c|}
\hline Inclusion criteria & Exclusion criteria \\
\hline - Age 18 or older & - Clinically significant suicide risk \\
\hline - Current major depression (DSM-IV-TR criteria) & - History of schizophrenia or psychotic symptoms \\
\hline - HAM-D total score $\geq 14$ & - Bipolar disorder \\
\hline $\begin{array}{l}\text { - Total ATHF Intensity score } \geq 5 \text { for } 2 \text { or more antidepressant } \\
\text { medications }\end{array}$ & - Obsessive compulsive disorder \\
\hline $\begin{array}{l}\text { - Evidence of two failed adequate antidepressant trials in the current } \\
\text { depressive episode }\end{array}$ & - Alcohol or substance abuse or dependence within the past 3 months \\
\hline \multirow[t]{5}{*}{ - Current use of antidepressants } & - Insufficient ability to understand or read English \\
\hline & - MMSE total score $<25$ \\
\hline & - Severe medical illness determined by the CIRS \\
\hline & $\begin{array}{l}\text { - History of meditation practice or currently meditation more than } 1 \text { time } \\
\text { per week }\end{array}$ \\
\hline & - Currently practicing yoga more than 2 times per week \\
\hline
\end{tabular}

Note. HAM-D = Hamilton Depression Rating Scale; ATHF = Antidepressant Treatment History Form; MMSE = Mini Mental Status Exam; CIRS = Cumulative IIIness Rating Scale.

${ }^{*}$ Axis I anxiety disorders are not excluded as they are highly co-morbid with depression. This study is designed to approximate a real world, clinical setting, and anxiety disorders were not excluded in the original MBCT studies nor in the STAR*D trials. 
$[33,34]$ to assess health status. Scores of 4 or more on any one item required further evaluation from the research team to ensure participants could perform the required physical activities in HEP. Table 4 outlines all measurements and a complete list of time points of administration.

Data obtained during the intake interview was presented in a weekly research conference where the principal investigator reviewed eligibility criteria on a case-by-case basis to make determinations about participants' eligibility for enrollment.

\section{Randomization and baseline assessment}

The unit of randomization was the patient, and randomization tables were stratified by gender to ensure balanced assignment. Patients were randomized during the baseline assessment, which occurred within two weeks of the commencement of treatment (see Figure 1. Study Design and Participant Flow). Baseline assessments served two purposes: 1 ) to ensure that patients continued to meet eligibility criteria for MDD with scores $\geq 14$ on the HAM-D and 2) to serve as the primary data collection point (see Outcome Measures section). Patients who reported improved depressive symptoms during the baseline interview with HAMD total scores $<14$, were not randomized to treatment and told that they could be reassessed for a future intervention cohort should their depression symptoms relapse. Participants who continued to be eligible on measures of depressive severity were then randomly assigned to either MBCT + TAU or HEP + TAU.

Table 4 Eligibility and outcome measures

\begin{tabular}{|c|c|c|c|c|c|c|c|c|c|c|c|c|c|}
\hline \multirow{2}{*}{$\begin{array}{l}\text { Category/ } \\
\text { Measure }\end{array}$} & \multicolumn{12}{|c|}{ Week of measurement point } & \multirow{2}{*}{$\begin{array}{l}\text { Method of } \\
\text { measurement* }\end{array}$} \\
\hline & \# Item & Screen & 0 & 1 & 2 & 3 & 4 & $5-7$ & 8 & 24 & 36 & 52 & \\
\hline Eligibility and demographic measures & & & & & & & & & & & & & $\mathrm{Cl}$ \\
\hline Contact InfolDemographic data & - & $x$ & & & & & & & & & & & $\mathrm{Cl}$ \\
\hline Mini mental status exam & 11 & $x$ & & & & & & & & & & & $\mathrm{Cl}$ \\
\hline Structured clinical interview for diagnosis of axis $1 / \|$ & - & $x$ & & & & & & & & & & & $\mathrm{Cl}$ \\
\hline Antidepressant treatment history form & - & $x$ & & & & & & & & & & & $\mathrm{Cl}$ \\
\hline Cumulative illness rating scale & & $x$ & & & & & & & & & & & $\mathrm{Cl}$ \\
\hline \multicolumn{14}{|l|}{ Depression outcomes } \\
\hline Hamilton depression rating scale & 17 & $x$ & $x$ & & & & $x$ & & $x$ & $x$ & $x$ & $x$ & $\mathrm{Cl}$ \\
\hline Quick inventory of depression symptomatology & 16 & & $x$ & $x$ & $x$ & $x$ & $x$ & $x$ & $x$ & $x$ & $x$ & $x$ & $\mathrm{PQO}$ \\
\hline Clinical global impressions scale & 2 & & $x$ & & & & $x$ & & $X$ & $x$ & $x$ & $x$ & $\mathrm{Cl}$ \\
\hline SCID mood module for major depression & - & & & & & & & & $X$ & $x$ & $x$ & $x$ & $\mathrm{Cl}$ \\
\hline Longitudinal interval follow-up evaluation & - & & & & & & & & $X$ & $x$ & $x$ & $x$ & $\mathrm{Cl}$ \\
\hline \multicolumn{14}{|l|}{ Functional status outcomes } \\
\hline Health status-short form 12 & 12 & & $x$ & & & & $x$ & & $X$ & $x$ & $x$ & $x$ & $\mathrm{PQO}$ \\
\hline Work and social activity scale & 5 & & $x$ & & & & $x$ & & $X$ & $X$ & $x$ & $x$ & $\mathrm{PQO}$ \\
\hline Quality of life enjoyment satisfaction questionnaire & 15 & & $x$ & & & & $x$ & & $X$ & $x$ & $x$ & $x$ & $\mathrm{PQO}$ \\
\hline \multicolumn{14}{|l|}{ Measures of mediation/moderation } \\
\hline Therapeutic rationale scale & 4 & & & & $x$ & & & & $x$ & & & & PQO \\
\hline State trait anxiety inventory & 40 & & $x$ & & & & $x$ & & $x$ & $x$ & $x$ & $x$ & $\mathrm{PQO}$ \\
\hline Perceived stress scale & 14 & & $x$ & & & & $x$ & & $X$ & $x$ & $x$ & $x$ & PQO \\
\hline Childhood trauma questionnaire & & $x$ & & & & & & & & & & & PQO \\
\hline Ruminative response scale & 22 & & $x$ & & & & $x$ & & $x$ & $X$ & $x$ & $x$ & PQO \\
\hline Acceptance and avoidance questionnaire & 10 & & $x$ & & & & $x$ & & $X$ & $x$ & $x$ & $x$ & PQO \\
\hline Five facet mindfulness questionnaire & 30 & & $x$ & & & & $x$ & & $x$ & $x$ & $x$ & $x$ & PQO \\
\hline Experiences questionnaire & 20 & & $x$ & & & & $x$ & & $X$ & $x$ & $x$ & $x$ & PQO \\
\hline Self-compassion scale & 26 & & $x$ & & & & $x$ & & $x$ & $x$ & $x$ & $x$ & $\mathrm{PQO}$ \\
\hline Medication/Psychotherapy change form & - & & $x$ & $x$ & $x$ & $x$ & $x$ & $x$ & $x$ & $x$ & $x$ & $x$ & $\mathrm{Cl}$ \\
\hline Homework summary log & - & & $x$ & & $x$ & $x$ & $x$ & $x$ & $x$ & $x$ & $x$ & $x$ & PQO \\
\hline
\end{tabular}

${ }^{*} \mathrm{Cl}=$ Clinical interview; $\mathrm{PQO}=$ Patient questionnaire online. 


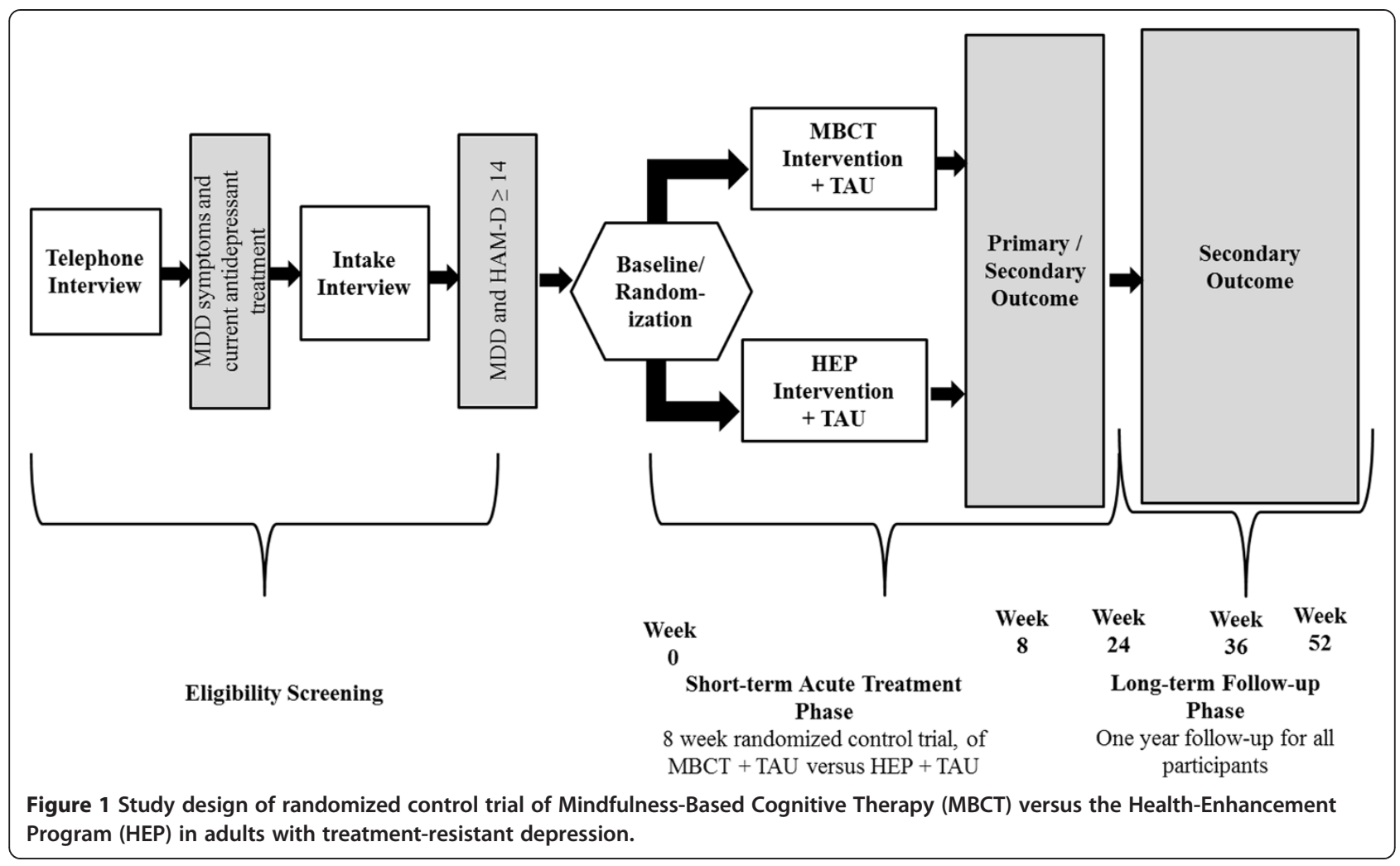

\section{Outcome measures}

Outcomes were assessed at baseline, weeks 1-7, at the end of treatment (week 8) and at weeks 24, 36 and 52 during the long-term follow-up phase. Data was obtained from patient questionnaires completed online and through clinical RA ratings, which were confirmed with chart review of medical records when necessitated.

\section{Primary and secondary outcome measures}

The primary outcome measurement was improvement of depression severity after 8 weeks, as measured through change in HAM-D total scores from pre- to post-treatment, treatment response (scores $\geq 50 \%$ decrease from baseline) and remission (post-treatment scores $\leq 7$ ). Length of treatment efficacy, a secondary outcome, was assessed up to 1 year after baseline using HAM-D scores, response and remission rates by measuring rate of depression relapse using the semi-structured Longitudinal Interval Follow-up Evaluation (LIFE) [35]. The participant-rated 16-item Quick Inventory of Depressive Symptomatology (QIDS) [36] was used as a secondary measurement to assess change in depressive severity, response (scores $\geq 50 \%$ decrease from baseline) and remission (scores $\leq 5$ ) during the same treatment and follow-up time period.

Functional status and quality of life were additional secondary outcomes measured using the SF-12 Health Survey [37,38], the Work and Social Adjustment Scale
(WSAS) [39], [40] and the Quality of Life, Enjoyment, and Satisfaction Questionnaire (Q-LES-SQ) [41].

\section{Mediators, moderators and potential covariates}

A number of other psychometric variables believed to be associated with mindfulness were investigated and measured at baseline, during mid-point (week 4), after the end of treatment (week 8) and the long-term follow-up weeks 24,36 , and 52 . The following potential mediators related to $\mathrm{MBCT}$ were measured: mindfulness with the Five Facet Mindfulness Questionnaire [42]; experiential avoidance with the Acceptance and Action Questionnaire (AAQ) [43]; rumination with the Ruminative Response Scale (RRS) [44,45]; and self-compassion with the Self-Compassion Scale (SCS) [46].

Psychiatric factors that may impact depression severity and resistance such as early age of depression onset, number of recurrences, poor treatment outcomes (e.g. incomplete remission) and co-morbid psychiatric illness (e.g. anxiety disorders [47] or personality disorders [48]), will be included in the analysis as covariates [49]. Also included will be other factors known to be moderators of depression treatment outcomes. These include demographic factors such as minority status, ethnicity and lower socio-economic status [50] and stress, both current $[51,52]$ and a childhood history of stress [53] (trauma, abuse, neglect) which were collected during the baseline assessment. 
Medication changes in the TAU arm are the same ones utilized in treating persisting or worsening depression in the STAR*D studies $[3,8]$. Changes were tracked during the 8-week treatment and over the 52 week follow-up through patient report and chart review. Alternate forms of treatment (i.e. individual psychotherapy, group psychotherapy, electroconvulsive therapy, etc.) for depression was tracked after the treatment-phase of the study through patient report and interview. These treatments will be analyzed as covariates as well as secondary outcomes, similar to the aforementioned medication changes.

Treatment expectancy to assess potential moderating effects of positive expectancy was measured through the Treatment Rationale Scale [54-56], which participants completed after the first and final sessions (weeks 2 and 8). Changes in psychotropic medications including antidepressants, benzodiazepines and mood stabilizers were tracked at every assessment time point using a medication evaluation form. Lastly, participant engagement in treatment was measured through attendance and total time spent completing homework and related activities outside of class. A complete list of the outcome measures, measurement points and assessment methods for the study are summarized in Table 4.

\section{Treatment adherence and fidelity assessment}

Adherence to treatment was defined by measuring the received therapy dose through number of sessions attended. In the current trial, a completer of treatment was defined as participation in at least five out of eight sessions.

Group leaders digitally recorded all treatment sessions, and recordings were sent to independent evaluators not affiliated with the university or part of the research team. MBCT adherence and competency was provided by an expert in MBCT who reviewed 3 randomly selected sessions from each treatment cohort. For the control condition, external monitoring of treatment HEP adherence and facilitator competency was completed by a HEP expert who also reviewed 3 randomly selected sessions from each treatment cohort. Internal treatment adherence and competency for both interventions was assessed by the principal investigator who reviewed the same 3 randomly selected sessions. The MBCT Adherence Scale [57], an instrument used in the original studies of MBCT, was used to assess treatment fidelity. Inter-rater reliability ranges from 0.59 for the Cognitive Therapy subscale and .97 for the Mindfulness subscale, and 0.82 for global ratings. Competence in delivering $\mathrm{MBCT}$ is rated with a newly developed measure, MBCT Competence Scale [13]. This 10-item instrument uses a scale from $0-5$ to assess each facilitator's skill in delivering the treatment. Treatment Fidelity in HEP was assessed using the HEP Adherence Scale. The HEP Adherence Scale is a newly developed scale and is modeled after the MBCT Adherence Scale. It is a 17-item instrument where items are score from 1 to 3 . Competence in delivering HEP was rated with a newly developed instrument, the HEP Competency Scale. This is a 10-point scale for rating group facilitator skill and expertise in delivering the intervention. Because the HEP Adherence Scale, HEP Competency Scale, and MBCT Competency Scale have not been previously validated, they will serve as templates for assessment in this study and pilot data for future research. Based on reviews and results of adherence and competency measures, feedback was provided to group leaders during weekly supervision meetings.

\section{Data safety and monitoring plan}

In consultation with NCCAM, an independent Data Safety Monitoring Board (DSMB) was established to oversee all study activities to ensure the safety of participants, the validity of our findings and the need for further data collection. Three categories of patient safety concerns were highlighted for this study: 1) increased depression severity; 2) risk to safety through self-harm and increased suicidal ideation and; 3) study withdrawals that were potentially or definitely related to specific factors in each treatment intervention. Regarding suicidal ideation among enrolled participants, when patients responded positively to the suicidality questions on the HAM-D and QIDS or experienced a 1 or greater point increase from their baseline scores, RAs initiated the following actions they: 1) they notified the PI; 2) provided patients with referrals to crisis hotlines; 3) discussed with patients whether the patient had notified their prescribing physician or mental health provider about their symptoms and; 4) in some cases, where the PI deemed that more clinical contact was warranted, they corresponded via telephone and/or sent confidential letters and email correspondences to providers notifying them of their clinical impressions.

\section{Planned analytic approach}

The primary outcome measure of the HAM-D will be compared between participants randomized to the MBCT + TAU and the HEP + TAU groups using a mixed-effects statistical model $[58,59]$. Mean levels of severity, and response and remission rates at week 8 and bivariate measures will be compared between treatment conditions using logistic regression models with generalized estimating equations to correct for interdependence resulting from group membership. The secondary outcome measures of the QIDS-SR and CGI will be compared using the same methodology but replacing the HAM-D with the QIDS-SR and CGI severity measures. We will also assess relapse occurrence in both arms using the LIFE interview at weeks 24,36 and 52. 
The efficacy of MBCT + TAU versus HEP + TAU on functional status as a secondary outcome will be assessed using similar models to the methods used to test the reduction in HAM-D 17 scores. For each of the four scales, scores at week 8 will be compared between randomized conditions using a mixed-effects statistical model, which will include effects for randomized condition, therapy group and baseline disability score. The test of the estimated parameter for treatment condition will be a direct test of this hypothesis. Mediating effects of the relationship of treatment assignment with outcome will be tested using a series of linear regression models. Putative mediators, enhanced mindfulness (FFMQ), enhanced selfcompassion (SCS), diminished rumination (RRS) and decreased experiential avoidance (AAQ) will be assessed at weeks 4, 8, 24, 36, and 52. We will also assess persistence of treatment changes in depression (HAM-D, QIDSSR, CGI), functional status (SF-12, WSAS, Q-LES-Q-SF), mindfulness (FFMQ), rumination (RRS), self-compassion (SCS) and avoidance (AAQ) at weeks 24, 36 and 52 by analyses parallel to those outlined for testing Hypotheses 1 and 2 using data from the weeks 24, 36, and Week 52. Mixed-effects and logistic regression models using a generalized estimating equation (GEE) will allow direct testing of these effects. Moderating factors (e.g. age of depression onset, prior poor treatment outcomes, co-morbid psychiatric diagnoses and other socio-demographics) associated with TRD will also be included in the model.

Additional secondary analyses will test for effects of time. These models will also include indicator variables of any additional treatments (e.g. HEP participants' crossing over to take MBCT outside of the research), medication changes after the end of acute treatment before the 24, 36 and 52-week follow-ups and also whether or not patients began new psychotherapeutic interventions after treatment or continued formal meditation practices.

Based on the extent and nature of missing data (outcomes, predictors, baseline, and items from scales), we will use one of several strategies for data analysis. We anticipate that the majority of missing data will result from participant attrition. In this case, we will know the randomization assignment and have data from some, but not all, of the planned assessments. Our primary approach to dealing with this monotone missing data pattern will be a patternmixture modeling approach [60]. We may also be missing some data on predictors, even in the absence of participant attrition. Our primary method for dealing with missing predictor values will be multiple imputation using a regression modeling approach [60]. This method is superior to simpler methods such as using group means for missing data. In order to limit attrition, we will provide an incentive in the form of an assessment payment schedule to increase motivation to complete all assessments.

\section{Discussion}

Treatment-resistant depression is a condition associated with high rates of disability, chronicity, reoccurrence and failed response to antidepressant treatment $[3,8]$. Through decreased workforce productivity and disability, the economic burden caused by refractory depression is enormous [10,61]; hence the importance of finding effective treatment modalities for this population is clear and of vital public health interest. This investigation represents the first RCT to evaluate MBCT for TRD. Results from this study will address whether MBCT is superior in reducing depression severity after 8 week treatment and improving relapse and remission rates over time in comparison to a credible comparator condition, developed to control for non-specific treatment effects. If successful, this study will identify an augmentation treatment for a large, hard-to-treat population for whom few treatments have been effective.

After analysis, we will be able to assess whether the specific constructs of a mindfulness-based intervention, such as the putative mediators in MBCT (increased mindfulness, self-compassion and decreased rumination and experiential avoidance) are enhanced more after an 8-week treatment with MBCT compared with HEP, and whether they are associated with positive treatment outcomes. We will also be able to evaluate whether treatment gains between groups (i.e. reduced depression severity, increased functional status) are retained during a 1-year follow-up and whether differential effects might be mediated by the specific constructs of MBCT listed above. Finally, we will be able to assess whether participant expectancy, treatment adherence (i.e. number of sessions attended), and compliance (i.e. number of minutes of homework completed) predicted treatment outcomes.

\section{Limitations}

The TAU intervention has some variance because we allowed medication changes to take place, which increases the possibility of wash-out effects of MBCT or falsely amplifying its effects. Moreover, antidepressants alone are also well documented to be associated with placebo effects, although these appear to be reduced in a TRD population [62]. Because our aim was to show that MBCT is an effective augmentation strategy and because we wanted trial results to be widely generalizable in light of routine clinical practice, we permitted some flexibility in medication changes. We anticipate that randomization and statistical analysis will control for this variance and allow us to compare the impact of medication changes in both arms.

The current investigation lacks a $3^{\text {rd }}$ arm or wait-list control condition, which limits the ability to determine whether factors unrelated to treatment, such as time or life events effects were associated with treatment effects. We will however, be able to compare our outcomes to 
similar TRD populations presented by Kenny and Williams [12] and Eisendrath et al., [13]. While we expect the association to be modest, we predict that treatment expectancies or patients' initial beliefs about the success of either intervention could influence treatment outcomes. The current investigation lacks a measure of treatment preference, which could have played a predictive role in affecting clinical outcomes.

\section{Summary}

In summary, the results of this trial will address whether MBCT, previously shown to be effective for relapse prevention, may be an effective augmentation therapy for patients with TRD in active depressive episodes. In addition, this is the first RCT to compare whether MBCT is more effective in reducing depression, rates of relapse and disability when augmented by medications (TAU) than an active comparator condition; thereby isolating the elements of MBCT that might be most salient. In particular, the study will highlight whether mindfulness or other features of the interventions play important roles in the outcomes. In addition, the study will shed light on which MBCT factors play mediating roles in producing its effects. National self-report surveys have found complementary and alternative techniques such as mindfulness, are being used by $41 \%$ to $54 \%$ [63] of individuals with depression, indicating that there is substantial public demand for mindfulness-based treatment interventions. Consequently we believe the results of this study will have significant applications for depression care by informing providers about targeted, population-based non-pharmacological interventions strategies that may also be appealing to TRD patients.

\section{Abbreviations \\ TRD: Treatment-resistant depression; MBCT: Mindfulness-based cognitive therapy; HEP: Health-Enhancement Program; TAU: Treatment as usual; CBT: Cognitive behavioral therapy; RA: Research assistant; \\ MBSR: Mindfulness-Based Stress Reduction; IRB: Institutional review board; PI: Principal investigator; DSMB: Data safety monitoring board; GEE: Generalized estimating equation.}

\section{Competing interests}

The authors declare that they have no competing interests.

\section{Authors' contributions}

SJE developed the original study design and secured funding. SJE and EPG drafted the primary manuscript. KLD contributed the sample size calculation and data analysis plan for reporting of study results. SJE, EPG, MC, JCS, DHM KLD, ZVS and MF participated in editing this paper. All authors substantially revised the article, read and approved the final manuscript.

\section{Acknowledgements}

This study is funded by the NIH/ National Center for Complementary and Alternative Medicine (NCCAM) grant number R01AT004572. The authors would like to gratefully thank Ms. Lauren Erickson, Christa Hogan, Natalie Holbrook and other members of the research staff who were responsible for subject recruitment, tracking/scheduling and data collection.

\section{Author details}

${ }^{1}$ University of California, San Francisco Department of Psychiatry, 401

Parnassus Avenue, Box 0984-AIP, San Francisco, CA 94143, USA. ${ }^{2}$ San
Francisco VA Medical Center, 4150 Clement Street, San Francisco, CA 94121, USA. ${ }^{3}$ University of Wisconsin, Madison Hospital \& Clinics Sports Medicine Center, 621 Science Drive, Madison, WI 53711, USA. ${ }^{4}$ Department of Psychiatry, University of Toronto, 250 College Street, Toronto, ON M5T1R8, USA. ${ }^{5}$ University of California, San Francisco, Division of General Internal Medicine, 1545 Divisadero Street, Suite 315, San Francisco, CA 94143-0320, USA.

Received: 3 February 2014 Accepted: 27 February 2014 Published: 11 March 2014

\section{References}

1. Burden of mental and behavioral disorders. In Volume The WHO Report 2001: Menatl Health, New Understanding, New Hope. Edited by Health M. New Hope: WHO; 2001

2. Murray CJ, Lopez AD: Evidence-based health policy-lessons from the Global Burden of Disease Study. Science 1996, 274(5288):740-743.

3. Rush AJ, Trivedi MH, Wisniewski SR, Stewart JW, Nierenberg AA, Thase ME, Ritz L, Biggs MM, Warden D, Luther JF, Shores-Wilson K, Niederehe G, Fava $M$; STAR*D Study Team: Bupropion-SR, sertraline, or venlafaxine-XR after failure of SSRIs for depression. N Engl J Med 2006, 354(12):1231-1242.

4. Greden JF: The burden of disease for treatment-resistant depression. J Clin Psychiatry 2001, 62(Suppl 16):26-31.

5. Fava M: Diagnosis and definition of treatment-resistant depression. Biol Psychiatry 2003, 53(8):649-659.

6. Quitkin FM, McGrath PJ, Stewart JW, Deliyannides D, Taylor BP, Davies CA, Klein DF: Remission rates with 3 consecutive antidepressant trials: effectiveness for depressed outpatients. J Clin Psychiatry 2005, 66(6):670-676.

7. Nierenberg AA, Feighner JP, Rudolph R, Cole JO, Sullivan J: Venlafaxine for treatment-resistant unipolar depression. J Clin Psychopharmacol 1994, 14(6):419-423.

8. Trivedi MH, Fava M, Wisniewski SR, Thase ME, Quitkin F, Warden D, Ritz L, Nierenberg AA, Lebowitz BD, Biggs MM, Luther JF, Shores-Wilson K, Rush AJ: Medication augmentation after the failure of SSRIs for depression. $N$ Engl J Med 2006, 354(12):1243-1252.

9. Wang PS, Berglund P, Olfson M, Pincus HA, Wells KB, Kessler RC: Failure and delay in initial treatment contact after first onset of mental disorders in the National Comorbidity Survey Replication. Arch Gen Psychiatry 2005, 62(6):603-613.

10. Kessler RC, Berglund P, Demler O, Jin R, Koretz D, Merikangas KR, Rush AJ, Walters EE, Wang PS: The epidemiology of major depressive disorder: Results From the National Comorbidity Survey Replication (NCS-R). JAMA 2003, 289(23):3095-3105.

11. Segal Z, Williams JM, Teasdale J: Mindfulness -based cognitive therapy for depression. New York: The Guilford press; 2002.

12. Kenny MA, Williams JM: Treatment-resistant depressed patients show a good response to Mindfulness-based Cognitive Therapy. Behav Res Ther 2007, 45(3):617-625.

13. Eisendrath SJ, Delucchi K, Bitner R, Fenimore P, Smit M, McLane M: Mindfulness-based cognitive therapy for treatment-resistant depression: a pilot study. Psychother Psychosom 2008, 77(5):319-320.

14. Teasdale JD, Segal ZV, Williams JM, Ridgeway VA, Soulsby JM, Lau MA: Prevention of relapse/recurrence in major depression by mindfulness-based cognitive therapy. J Consult Clin Psychol 2000, 68(4):615-623.

15. Ma SH, Teasdale JD: Mindfulness-based cognitive therapy for depression: replication and exploration of differential relapse prevention effects. J Consult Clin Psychol 2004, 72(1):31-40.

16. Segal ZV, Kennedy S, Gemar M, Hood K, Pedersen R, Buis T: Cognitive reactivity to sad mood provocation and the prediction of depressive relapse. Arch Gen Psychiatry 2006, 63(7):749-755.

17. Omidi A, Mohammadkhani P, Mohammadi A, Zargar F: Comparing mindfulness based cognitive therapy and traditional cognitive behavior therapy with treatments as usual on reduction of major depressive disorder symptoms. Iran Red Crescent Med J 2013, 15(2):142-146.

18. Teasdale JD, Segal Z, Williams JM: How does cognitive therapy prevent depressive relapse and why should attentional control (mindfulness) training help? Behav Res Ther 1995, 33(1):25-39.

19. MacCoon DLA, Rosenkranz M, Davidson R: Lessons from an Active Control Condition: "McMindfulness, Shams, and the One-Fold Path. In Mind Life Summer Research Institute. New York: Garrison; 2007. 
20. MacCoon DG, Imel ZE, Rosenkranz MA, Sheftel JG, Weng HY, Sullivan JC, Bonus KA, Stoney CM, Salomons TV, Davidson RJ, Lutz A: The validation of an active control intervention for Mindfulness Based Stress Reduction (MBSR). Behav Res Ther 2012, 50(1):3-12.

21. Miller $L$, Weissman $M$ : Interpersonal psychotherapy delivered over the telephone to recurrent depressives, A pilot study. Depress Anxiety 2002, 16(3):114-117.

22. Mynors-Wallis LM, Gath DH, Day A, Baker F: Randomised controlled trial of problem solving treatment, antidepressant medication, and combined treatment for major depression in primary care. BMG 2000, 320(7226):26-30.

23. Baer RA: Mindfulness training as a clinical intervention: a conceptual and empirical review. Clinical Psychology: Science and Practice 2003, 10(2):125-143.

24. Depression Guideline Panel: Depression in primary care: volume 2, Treatment of Major Depression. Rockville, MD: US Department of Health and Human Serviced, Public Health Service, Angency for Health Care Policy and Research, AHCPR Publication No. 93-0551, April 1993; 1993.

25. Finucane A, Mercer SW: An exploratory mixed methods study of the acceptability and effectiveness of Mindfulness-Based Cognitive Therapy for patients with active depression and anxiety in primary care. BMC Psychiatry 2006, 6:14

26. Kingston J, Chadwick P, Meron D, Skinner TC: A pilot randomized control trial investigating the effect of mindfulness practice on pain tolerance, psychological well-being, and physiological activity. J Psychosom Res 2007, 62(3):297-300.

27. Donner A, Shoukri MM, Klar N, Bartfay E: Testing the equality of two dependent kappa statistics. Stat Med 2000, 19(3):373-387.

28. Hamilton M: Development of a rating scale for primary depressive illness. Br J Soc Clin Psychol 1967, 6(4):278-296.

29. First MB, Spitzer, Robert L, Gibbon M, Williams, Janet BW: Structured Clinical Interview for DSM-IV-TR Axis I Disorders, Research Version, Patient Edition (SCID-I/P). In Biometrics Research, New York State Psychiatric Institute; 2002

30. Guy W: ECDEU Assessment Manual for Psychopharmacology Revised (DHEW Publ No ADM 76-338). Rockville, MD: U.S. Dept. Health, Education and Welfare, Public Health Service, Alcohol, Drug Abuse and Mental Health Administration, NIMH Psychopharmacology Research Branch, Division of Extramural Research Programs; 1976.

31. Folstein MF, Folstein SE, McHugh PR: Mini-mental state, A practical method for grading the cognitive state of patients for the clinician. J Psychiatr Res 1975, 12(3):189-198.

32. Dew RE, Kramer SI, McCall WV: Adequacy of antidepressant treatment by psychiatric residents: the antidepressant treatment history form as a possible assessment tool. Acad Psychiatry 2005, 29(3):283-288.

33. Linn BS, Linn MW, Gurel L: Cumulative illness rating scale. J Am Geriatr Soc 1968, 16(5):622-626.

34. Miller MD, Paradis CF, Houck PR, Mazumdar S, Stack JA, Rifai AH, Mulsant B, Reynolds CF 3rd: Rating chronic medical illness burden in geropsychiatric practice and research: application of the Cumulative Illness Rating Scale. Psychiatry Res 1992, 41(3):237-248

35. Keller MB, Lavori PW, Friedman B, Nielsen E, Endicott J, McDonald-Scott P, Andreasen NC: The Longitudinal Interval Follow-up Evaluation, A comprehensive method for assessing outcome in prospective longitudinal studies. Arch Gen Psychiatry 1987, 44(6):540-548.

36. Rush AJ, Trivedi MH, Ibrahim HM, Carmody TJ, Arnow B, Klein DN, Markowitz JC, Ninan PT, Kornstein S, Manber R, Thase ME, Kocsis JH, Keller $M B$ : The 16-Item Quick Inventory of Depressive Symptomatology (QIDS), clinician rating (QIDS-C), and self-report (QIDS-SR): a psychometric evaluation in patients with chronic major depression. Biol Psychiatry 2003, 54(5):573-583.

37. Ware JE Jr, Sherbourne CD: The MOS 36-item short-form health survey (SF-36), I. Conceptual framework and item selection. Med Care 1992, 30(6):473-483.

38. Ware J Jr, Kosinski M, Keller SD: A 12-Item Short-Form Health Survey: construction of scales and preliminary tests of reliability and validity. Med Care 1996, 34(3):220-233.

39. Mundt JC, Marks IM, Shear MK, Greist JH: The Work and Social Adjustment Scale: a simple measure of impairment in functioning. Br J Psychiatry 2002, 180:461-464.

40. Cohen S, Kamarck T, Mermelstein R: A global measure of perceived stress. $J$ Health Soc Behav 1983, 24(4):385-396.

41. Endicott J, Nee J, Harrison W, Blumenthal R: Quality of Life Enjoyment and Satisfaction Questionnaire: a new measure. Psychopharmacol Bull 1993, 29(2):321-326
42. Baer RA, Smith GT, Hopkins J, Krietemeyer J, Toney L: Using self-report assessment methods to explore facets of mindfulness. Assessment 2006, 13(1):27-45

43. Hayes SC, Strosahl K, Wilson KG, Bissett RT, Pistorello J, Toarmino D, Polusny MA, Dykstra TA, Batten SV, Bergan J, Stewart SH, Zvolensky MJ, Eifert GH, Bond FW, Forsyth JP, Karekla M, Mccurry SM: Measuring experiential avoidance: a preliminary test of a working model. Psychol Bull 2004, 54:553-578

44. Nolen-Hoeksema S, Morrow J: A prospective study of depression and posttraumatic stress symptoms after a natural disaster: the 1989 Loma Prieta Earthquake. J Pers Soc Psychol 1991, 61(1):115-121.

45. Nolen-Hoeksema S, Parker LE, Larson J: Ruminative coping with depressed mood following loss. J Pers Soc Psychol 1994, 67(1):92-104.

46. Neff KD: The development and validation of a scale to measure self-compassion. Self and Identity 2003, 2(3):223-250.

47. Pollack MH: Comorbid anxiety and depression. J Clin Psychiatry 2005, 66(Suppl 8):22-29.

48. Souery D, Oswald P, Massat I, Bailer U, Bollen J, Demyttenaere K, Kasper S, Lecrubier Y, Montgomery S, Serretti A, Zohar J, Mendlewicz J: Clinical factors associated with treatment resistance in major depressive disorder: results from a European multicenter study. J Clin Psychiatry 2007, 68(7):1062-1070.

49. Rush AJ, Zimmerman M, Wisniewski SR, Fava M, Hollon SD, Warden D, Biggs MM, Shores-Wilson K, Shelton RC, Luther JF, Thomas B, Trivedi MH: Comorbid psychiatric disorders in depressed outpatients: demographic and clinical features. J Affect Disord 2005, 87(1):43-55.

50. Trivedi MH, Rush AJ, Wisniewski SR, Warden D, McKinney W, Downing M, Berman SR, Farabaugh A, Luther JF, Nierenberg AA, Callan JA, Sackeim HA: Factors associated with health-related quality of life among outpatients with major depressive disorder: a STAR*D report. J Clin Psychiatry 2006, 67(2):185-195.

51. Ravindran AV, Matheson K, Griffiths J, Merali Z, Anisman H: Stress, coping, uplifts, and quality of life in subtypes of depression: a conceptual frame and emerging data. J Affect Disord 2002, 71(1-3):121-130.

52. Bertschy G: [Resistant depression]. Rev Med Suisse 2007, 3(125):2072-2074. 2076-2077.

53. Nemeroff CB, Heim CM, Thase ME, Klein DN, Rush AJ, Schatzberg AF, Ninan PT, McCullough JP Jr, Weiss PM, Dunner DL, Rothbaum BO, Kornstein S, Keitner G, Keller MB: Differential responses to psychotherapy versus pharmacotherapy in patients with chronic forms of major depression and childhood trauma. Proc Natl Acad Sci USA 2003, 100(24):14293-14296.

54. Borkovec T: Effects of expectancy on the outcome of systematic desensitization and implosive treatments for analogue anxiety. Behav Ther 1972, 3:29-40.

55. Devilly GJB, Thomas D: Psychometric properties of the credibility/ expectancy questionnaire. J Behav Ther Exp Psychiatry 2000, 31(2):73-86.

56. Borkovec TDNS: Credibility of analogue therapy rationales. J Behav Therapy Experimental Psychiatry 1972, 3:257-260.

57. Segal Z, Teasdale J, Williams JM, Gemar M: The mindfulness-based cognitive therapy adherence scale: inter-rater reliability, adherence to protocol and treatment distinctiveness. Clinical Psychology and Psychotherapy 2002, 9:131-138.

58. Laird NM, Ware JH: Random-effects models for longitudinal data. Biometrics 1982, 38(4):963-974.

59. Cnaan A, Laird NM, Slasor P: Using the general linear mixed model to analyse unbalanced repeated measures and longitudinal data. Stat Med 1997, 16(20):2349-2380.

60. Little JM: Presenting statistics. Aust N Z J Surg 1987, 57(7):417-419.

61. Greenberg PE, Leong SA, Birnbaum HG, Robinson RL: The economic burden of depression with painful symptoms. J Clin Psychiatry 2003, 64(Suppl 7):17-23.

62. Quitkin FM: Placebos, drug effects, and study design: a clinician's guide. Am J Psychiatry 1999, 156(6):829-836.

63. Kessler RCSJ, Davis RB, Foster DF, Wilkey SA, Van Rompay MI, Eisenberg DM: The use of complementary and alternative therapies to treat anxiety and depression in the United States. Am J Psychiatry 2001, 158:289-294.

doi:10.1186/1472-6882-14-95

Cite this article as: Eisendrath et al:: Mindfulness-based cognitive therapy (MBCT) versus the health-enhancement program (HEP) for adults with treatment-resistant depression: a randomized control trial study protocol. BMC Complementary and Alternative Medicine 2014 14:95. 